\title{
An Extension of the Optimal Homotopy Asymptotic Method to Coupled Schrödinger-KdV Equation
}

\author{
Hakeem Ullah, Saeed Islam, Muhammad Idrees, Mehreen Fiza, and Zahoor Ul Haq \\ Department of Mathematics, Abdul Wali Khan University Mardan, 23200, Pakistan \\ Correspondence should be addressed to Hakeem Ullah; hakeemullah1@gmail.com
}

Received 26 December 2013; Accepted 13 March 2014; Published 7 May 2014

Academic Editor: Patricia J. Y. Wong

Copyright ( 2014 Hakeem Ullah et al. This is an open access article distributed under the Creative Commons Attribution License, which permits unrestricted use, distribution, and reproduction in any medium, provided the original work is properly cited.

\begin{abstract}
We consider the approximate solution of the coupled Schrödinger-KdV equation by using the extended optimal homotopy asymptotic method (OHAM). We obtained the extended OHAM solution of the problem and compared with the exact, variational iteration method (VIM) and homotopy perturbation method (HPM) solutions. The obtained solution shows that extended OHAM is effective, simpler, easier, and explicit and gives a suitable way to control the convergence of the approximate solution.
\end{abstract}

\section{Introduction}

The nonlinear Schrödinger equations are of great interest due to their numerous applications in physical phenomena. The coupled Schrödinger-KdV equations are extensively used to model nonlinear dynamics of one-dimensional Langmuir and ion acoustic waves in the system of coordinates moving at the speed of ion acoustic. This problem remains under consideration from many years and has been investigated by many researchers. Many authors have investigated the nonlinear Schrödinger-KdV equation by various techniques such as the following: Wang [1] used finite difference method, Küçükarslan [2] used HPM, Bai and Zhang [3] used quadratic B-Spline finite element method, Fan and Hon [4] used extended tanh method, Kaya and El-Sayed [5] used adomian decomposition method (ADM), Doosthoseini and Shahmohamadi [6] used VIM, Alomari et al. [7] used homotopy analysis method (HAM), Qing et al. [8] used element free Galerkin method (EFG), and Golbabai and Safdari-Vaighani [9] used meshless method using RBF collocation scheme. The perturbation methods like HPM required a small parameter and are difficult to determine.

Recently, Marinca et al. introduced OHAM [10-14] for the solution of nonlinear problems which made the perturbation methods independent of the assumption of small parameters and huge computational work.
The motivation of this paper is to extend the OHAM formulation for a system of three partial differential equations and to apply the extended OHAM formulation to coupled nonlinear Schrödinger-KdV equation. In [15-17] OHAM has been proved to be valuable for obtaining an approximate solution of ordinary/partial differential equations (O/PDEs). Before, this system of nonlinear partial differential equations (NPDEs) was not solved by OHAM. We have proved that extended OHAM is useful and reliable for NPDEs, showing its validity and great potential for the solution of transient physical phenomenon in science and engineering.

In the succeeding section, the basic idea of extended OHAM is formulated for the solution of system NPDEs. The effectiveness and efficiency of OHAM are shown in Section 3.

\section{Extended Mathematical Formulation of OHAM}

Consider a system of three partial differential equations:

$$
\begin{aligned}
\mathscr{A}_{1}(f(x, t))+s_{1}(x, t) & =0, \\
\mathscr{A}_{2}(g(x, t))+s_{2}(x, t) & =0, \\
\mathscr{A}_{3}(h(x, t))+s_{2}(x, t) & =0, \\
x & \in \Omega
\end{aligned}
$$




$$
\begin{aligned}
\mathscr{B}_{1}\left(f, \frac{\partial f}{\partial x}\right) & =0, \\
\mathscr{B}_{2}\left(g, \frac{\partial g}{\partial x}\right) & =0, \\
\mathscr{B}_{3}\left(h, \frac{\partial h}{\partial x}\right) & =0, \\
x & \in \Gamma,
\end{aligned}
$$

where $\mathscr{A}_{1}, \mathscr{A}_{2}, \mathscr{A}_{3}$ are differential operators, $f(x, t), g(x, t)$, $h(x, t)$ are unknown functions, $x$ and $t$ denote spatial and temporal independent variables, respectively, $\Gamma$ is the boundary of $\Omega$, and $s_{1}(x, t), s_{2}(x, t), s_{3}(x, t)$ are known analytic functions. $\mathscr{A}_{1}, \mathscr{A}_{2}, \mathscr{A}_{3}$ can be divided into two parts:

$$
\begin{aligned}
& \mathscr{A}_{1}=\mathscr{L}_{1}+\mathscr{N}_{1}, \\
& \mathscr{A}_{2}=\mathscr{L}_{2}+\mathscr{N}_{2}, \\
& \mathscr{A}_{3}=\mathscr{L}_{3}+\mathscr{N}_{3} .
\end{aligned}
$$

$\mathscr{L}_{1}, \mathscr{L}_{2}, \mathscr{L}_{3}$ contain the linear parts while $\mathcal{N}_{1}, \mathcal{N}_{2}, \mathcal{N}_{3}$ contain the nonlinear parts of the system of partial differential equations.

According to OHAM, we construct

$$
\begin{aligned}
& \alpha(x, t ; p): \Omega \times[0,1] \longrightarrow \mathfrak{R}, \\
& \beta(x, t ; p): \psi \times[0,1] \longrightarrow \mathfrak{R}, \\
& \gamma(x, t ; p): \phi \times[0,1] \longrightarrow \mathfrak{R},
\end{aligned}
$$

satisfying the following homotopies:

$$
\begin{aligned}
& H(\alpha(x, t ; p), p) \\
& =(1-p)\left\{\mathscr{L}_{1}(\alpha(x, t ; p))+s_{1}(x, t)\right\} \\
& \quad-H_{1}(p)\left\{\mathscr{A}_{1}(\alpha(x, t ; p))+s_{1}(x, t)\right\}=0, \\
& H(\beta(x, t ; p), p) \\
& =(1-p)\left\{\mathscr{L}_{2}(\beta(x, t ; p))+s_{2}(x, t)\right\} \\
& \quad-H_{2}(p)\left\{\mathscr{A}_{2}(\beta(x, t ; p))+s_{2}(x, t)\right\}=0, \\
& H(\gamma(x, t ; p), p) \\
& =(1-p)\left\{\mathscr{L}_{3}(\gamma(x, t ; p))+s_{3}(x, t)\right\} \\
& \quad-H_{3}(p)\left\{\mathscr{A}_{3}(\gamma(x, t ; p))+s_{3}(x, t)\right\}=0,
\end{aligned}
$$

where the auxiliary functions $H_{1}(p), H_{2}(p), H_{3}(p)$ are nonzero for $p \neq 0$ and $H_{1}(0)=0, H_{2}(0)=0, H_{3}(0)=0$.
Equation (4) is called optimal homotopy equation. Clearly, we have

$$
\begin{aligned}
p=0 \Longrightarrow & H(\alpha(x, t ; 0), 0)=\mathscr{L}_{1}(\alpha(x, t ; 0))+s_{1}(x, t)=0, \\
p=0 \Longrightarrow & H(\beta(x, t ; 0), 0)=\mathscr{L}_{2}(\beta(x, t ; 0))+s_{2}(x, t)=0, \\
p=0 \Longrightarrow & H(\gamma(x, t ; 0), 0)=\mathscr{L}_{3}(\gamma(x, t ; 0))+s_{3}(x, t)=0, \\
p & =1 \Longrightarrow H(\alpha(x, t ; 1), 1) \\
& =H_{1}(1)\left\{\mathscr{A}_{1}(\alpha(x, t ; p))+s_{1}(x, t)\right\}=0, \\
p & =1 \Longrightarrow H(\beta(x, t ; 1), 1) \\
& =H_{2}(1)\left\{\mathscr{A}_{2}(\beta(x, t ; p))+s_{2}(x, t)\right\}=0, \\
p & =1 \Longrightarrow H(\gamma(x, t ; 1), 1) \\
& =H_{3}(1)\left\{\mathscr{A}_{3}(\gamma(x, t ; p))+s_{3}(x, t)\right\}=0 .
\end{aligned}
$$

Obviously, when $p=0$ and $p=1$ we obtain

$$
\begin{aligned}
\alpha(x, t ; 0) & =f_{0}(x, t), \beta(x, t ; 0) \\
& =g_{0}(x, t), \gamma(x, t ; 0)=h_{0}(x, t), \\
\alpha(x, t ; 1) & =f(x, t), \beta(x, t ; 1) \\
& =g(x, t), \gamma(x, t ; 1)=h(x, t),
\end{aligned}
$$

respectively. When $p$ varies from 0 to 1 , the solution $\alpha(x, t ; p), \beta(x, t ; p), \gamma(x, t ; p)$ approaches from $f_{0}(x, t), g_{0}(x, t), h_{0}(x, t)$ to $f(x, t), g(x, t), h(x, t)$, where $f_{0}(x, t), g_{0}(x, t), h_{0}(x, t)$ are obtained from (4) for $p=0$ :

$$
\begin{array}{ll}
\mathscr{L}_{1}\left(f_{0}(x, t)\right)+s_{1}(x, t)=0, & \mathscr{B}_{1}\left(f_{0}, \frac{\partial f_{0}}{\partial x}\right)=0, \\
\mathscr{L}_{2}\left(g_{0}(x, t)\right)+s_{2}(x, t)=0, & \mathscr{B}_{2}\left(g_{0}, \frac{\partial g_{0}}{\partial x}\right)=0, \\
\mathscr{L}_{3}\left(h_{0}(x, t)\right)+s_{3}(x, t)=0, & \mathscr{B}_{3}\left(h_{0}, \frac{\partial h_{0}}{\partial x}\right)=0 .
\end{array}
$$

We choose auxiliary functions $H_{1}(p), H_{2}(p), H_{3}(p)$ in the form

$$
\begin{aligned}
& H_{1}(p)=p C_{11}+p^{2} C_{12}+p^{3} C_{13}+\cdots+p^{m} C_{1 m} \\
& H_{2}(p)=p C_{21}+p^{2} C_{22}+p^{3} C_{23}+\cdots+p^{m} C_{2 m} \\
& H_{3}(p)=p C_{31}+p^{2} C_{32}+p^{3} C_{33}+\cdots+p^{m} C_{3 m} .
\end{aligned}
$$

To get the approximate solutions, we expand $\alpha\left(x, t ; p, C_{1 i}\right), \beta\left(x, t ; p, C_{2 i}\right), \gamma\left(x, t ; p, C_{3 i}\right)$ by Taylor's series about $p$ in the following manner:

$$
\begin{aligned}
& \alpha\left(x, t ; p, C_{1 i}\right)=f_{0}(x, t)+\sum_{k \geq 1} f_{k}\left(x, t ; C_{1 i}\right) p^{k}, \\
& \beta\left(x, t ; p, C_{2 i}\right)=g_{0}(x, t)+\sum_{l \geq 1} g_{l}\left(x, t ; C_{2 i}\right) p^{k}, \\
& \gamma\left(x, t ; p, C_{3 i}\right)=h_{0}(x, t)+\sum_{n \geq 1} h_{n}\left(x, t ; C_{3 i}\right) p^{k},
\end{aligned}
$$


where $k=l=n=i=1,2,3,4, \ldots$ Now substituting (8)(9) into (4) and equating the coefficient of like powers of $p$, we obtain zeroth order system, given by (7) and the first and second order systems given by (10)-(11), respectively, and the general governing equations for $u_{k}(x, t)$ are given by (12):

$$
\begin{aligned}
& \mathscr{L}_{1}\left(f_{1}(x, t)\right)-\mathscr{L}_{1}\left(f_{0}(x, t)\right) \\
& =C_{11}\left(\mathscr{L}_{1}\left(f_{0}(x, t)\right)+\mathscr{N}_{1}\left(f_{0}(x, t)\right)\right), \\
& \mathscr{B}_{1}\left(f_{1}, \frac{\partial f_{1}}{\partial x}\right)=0 \\
& \mathscr{L}_{2}\left(g_{1}(x, t)\right)-\mathscr{L}_{2}\left(g_{0}(x, t)\right) \\
& =C_{21}\left(\mathscr{L}_{2}\left(g_{0}(x, t)\right)+\mathcal{N}_{2}\left(g_{0}(x, t)\right)\right), \\
& \mathscr{B}_{2}\left(g_{1}, \frac{\partial g_{1}}{\partial x}\right)=0 \\
& \mathscr{L}_{3}\left(h_{1}(x, t)\right)-\mathscr{L}_{3}\left(h_{0}(x, t)\right) \\
& =C_{31}\left(\mathscr{L}_{3}\left(h_{0}(x, t)\right)+\mathcal{N}_{3}\left(h_{0}(x, t)\right)\right) \text {, } \\
& \mathscr{B}_{3}\left(h_{1}, \frac{\partial h_{1}}{\partial x}\right)=0 \\
& \mathscr{L}_{1}\left(f_{2}(x, t)\right)-\mathscr{L}_{1}\left(f_{1}(x, t)\right) \\
& =C_{11}\left(\mathscr{L}_{1}\left(f_{1}(x, t)\right)+\mathcal{N}_{1}\left(f_{0}(x, t), f_{1}(x, t)\right)\right) \\
& +C_{12}\left(\mathscr{L}_{1}\left(f_{0}(x, t)\right)+\mathcal{N}_{1}\left(f_{0}(x, t)\right)\right) \text {, } \\
& \mathscr{B}_{1}\left(f_{2}, \frac{\partial f_{2}}{\partial x}\right)=0, \\
& \mathscr{L}_{2}\left(g_{2}(x, t)\right)-\mathscr{L}_{2}\left(g_{1}(x, t)\right) \\
& =C_{21}\left(\mathscr{L}_{2}\left(g_{1}(x, t)\right)+\mathcal{N}_{2}\left(g_{0}(x, t), g_{1}(x, t)\right)\right) \\
& +C_{22}\left(\mathscr{L}_{2}\left(g_{0}(x, t)\right)+\mathscr{N}_{2}\left(g_{0}(x, t)\right)\right), \\
& \mathscr{B}_{2}\left(g_{2}, \frac{\partial g_{2}}{\partial x}\right)=0, \\
& \mathscr{L}_{3}\left(h_{2}(x, t)\right)-\mathscr{L}_{3}\left(h_{1}(x, t)\right) \\
& =C_{31}\left(\mathscr{L}_{3}\left(h_{1}(x, t)\right)+\mathcal{N}_{3}\left(h_{0}(x, t), h_{1}(x, t)\right)\right) \\
& +C_{32}\left(\mathscr{L}_{3}\left(h_{0}(x, t)\right)+\mathcal{N}_{3}\left(h_{0}(x, t)\right)\right) \text {, } \\
& \mathscr{B}_{3}\left(h_{2}, \frac{\partial h_{2}}{\partial x}\right)=0, \\
& \mathscr{L}_{1}\left(f_{3}(x, t)\right)-\mathscr{L}_{1}\left(f_{2}(x, t)\right) \\
& =C_{11}\left(\mathscr{L}_{1}\left(f_{2}(x, t)\right)\right. \\
& \left.+\mathcal{N}_{1}\left(f_{0}(x, t), f_{1}(x, t), f_{2}(x, t)\right)\right) \\
& +C_{12}\left(\mathscr{L}_{1}\left(f_{1}(x, t)\right)+\mathscr{N}_{1}\left(f_{0}(x, t), f_{1}(x, t)\right)\right) \\
& +C_{13}\left(\mathscr{L}_{1}\left(f_{0}(x, t)\right)+\mathscr{N}_{1}\left(f_{0}(x, t)\right)\right)
\end{aligned}
$$

(11)

$$
\begin{gathered}
\mathscr{B}_{1}\left(f_{3}, \frac{\partial f_{3}}{\partial x}\right)=0, \\
\mathscr{L}_{2}\left(g_{3}(x, t)\right)-\mathscr{L}_{2}\left(g_{2}(x, t)\right) \\
=C_{21}\left(\mathscr{L}_{2}\left(g_{2}(x, t)\right)\right. \\
\left.+\mathcal{N}_{2}\left(g_{0}(x, t), g_{1}(x, t), g_{2}(x, t)\right)\right) \\
+C_{22}\left(\mathscr{L}_{2}\left(g_{1}(x, t)\right)+\mathcal{N}_{2}\left(g_{0}(x, t), g_{1}(x, t)\right)\right) \\
+C_{23}\left(\mathscr{L}_{2}\left(g_{0}(x, t)\right)+\mathcal{N}_{2}\left(g_{0}(x, t)\right)\right), \\
\mathscr{B}_{2}\left(g_{3}, \frac{\partial g_{3}}{\partial x}\right)=0, \\
\mathscr{L}_{3}\left(h_{3}(x, t)\right)-\mathscr{L}_{3}\left(h_{2}(x, t)\right) \\
=C_{31}\left(\mathscr{L}_{3}\left(h_{2}(x, t)\right)\right. \\
\left.+\mathcal{N}_{3}\left(h_{0}(x, t), h_{1}(x, t), h_{2}(x, t)\right)\right) \\
+C_{32}\left(\mathscr{L}_{3}\left(h_{1}(x, t)\right)+\mathcal{N}_{3}\left(h_{0}(x, t), h_{1}(x, t)\right)\right) \\
+C_{33}\left(\mathscr{L}_{3}\left(h_{0}(x, t)\right)+\mathcal{N}_{3}\left(h_{0}(x, t)\right)\right), \\
\mathscr{B}_{3}\left(h_{3}, \frac{\partial h_{3}}{\partial x}\right)=0,
\end{gathered}
$$$$
\mathscr{L}_{1}\left(f_{k}(x, t)\right)-\mathscr{L}_{1}\left(f_{k-1}(x, t)\right)
$$$$
=\sum_{i=1}^{k} C_{1 i}\left[\mathscr{L}_{1}\left(f_{k-i}(x, t)\right)\right.
$$$$
\left.+\mathcal{N}_{1}\left(f_{0}(x, t), f_{1}(x, t), \ldots, f_{k-i}(x, t)\right)\right],
$$$$
k=2,3, \ldots \text {, }
$$$$
\mathscr{B}_{1}\left(f_{k}, \frac{\partial f_{k}}{\partial x}\right)=0,
$$$$
\mathscr{L}_{2}\left(g_{k}(x, t)\right)-\mathscr{L}_{2}\left(g_{k-1}(x, t)\right)
$$$$
=\sum_{i=1}^{k} C_{2 i}\left[\mathscr{L}_{2}\left(g_{k-i}(x, t)\right)\right.
$$$$
\left.+\mathcal{N}_{2}\left(g_{0}(x, t), g_{1}(x, t), \ldots, g_{k-i}(x, t)\right)\right]
$$$$
k=2,3, \ldots \text {, }
$$

$$
\begin{gathered}
\mathscr{B}_{2}\left(g_{k}, \frac{\partial g_{k}}{\partial x}\right)=0, \\
\mathscr{L}_{3}\left(h_{k}(x, t)\right)-\mathscr{L}_{3}\left(h_{k-1}(x, t)\right) \\
=\sum_{i=1}^{k} C_{3 i}\left[\mathscr{L}_{3}\left(h_{k-i}(x, t)\right)\right. \\
\left.+\mathcal{N}_{3}\left(h_{0}(x, t), h_{1}(x, t), \ldots, h_{k-i}(x, t)\right)\right], \\
\mathscr{B}_{3}\left(h_{k}, \frac{\partial h_{k}}{\partial x}\right)=0 .
\end{gathered}
$$


It has been observed that the convergence of the series (9) depends upon the auxiliary constants $C_{11}, C_{12}, C_{13}, \ldots C_{21}$, $C_{22}, C_{23}, \ldots C_{31}, C_{32}, C_{33}, \ldots$. If it is convergent at $p=1$, one has

$$
\begin{array}{r}
\alpha^{*}\left(x, t ; C_{1 i}\right)=f_{0}(x, t)+\sum_{k \geq 1} f_{k}\left(x, t ; C_{1 i}\right), \\
\beta^{*}\left(x, t ; C_{2 i}\right)=g_{0}(x, t)+\sum_{l \geq 1} g_{l}\left(x, t ; C_{2 i}\right), \\
i=1,2, \ldots m \\
\gamma^{*}\left(x, t ; C_{3 i}\right)=h_{0}(x, t)+\sum_{n \geq 1} h_{n}\left(x, t ; C_{3 i}\right) .
\end{array}
$$

Substituting (14) into (1.1), the following expression for residuals results:

$$
\begin{aligned}
R_{1}\left(x, t ; C_{1 i}\right)= & \mathscr{L}_{1}\left(\alpha^{*}\left(x, t ; C_{1 i}\right)\right) \\
& +s_{1}(x, t)+\mathcal{N}_{1}\left(\alpha^{*}\left(x, t ; C_{1 i}\right)\right), \\
R_{2}\left(x, t ; C_{2 i}\right)= & \mathscr{L}_{2}\left(\beta^{*}\left(x, t ; C_{2 i}\right)\right) \\
& +s_{2}(x, t)+\mathcal{N}_{2}\left(\beta^{*}\left(x, t ; C_{2 i}\right)\right), \\
R_{3}\left(x, t ; C_{3 i}\right)= & \mathscr{L}_{3}\left(\gamma^{*}\left(x, t ; C_{3 i}\right)\right) \\
& +s_{3}(x, t)+\mathcal{N}_{3}\left(\gamma^{*}\left(x, t ; C_{3 i}\right)\right) .
\end{aligned}
$$

If $R_{1}\left(x, t ; C_{1 i}\right)=0, R_{2}\left(x, t ; C_{2 i}\right)=0, R_{3}\left(x, t ; C_{3 i}\right)=0$ then $\alpha^{*}\left(x, t ; C_{1 i}\right), \beta^{*}\left(x, t ; C_{2 i}\right)$, and $\gamma^{*}\left(x, t ; C_{3 i}\right)$ will be the exact solutions of the problem. Generally it does not happen, especially in nonlinear problems.

For the computation of auxiliary constants, $C_{1 i}, C_{2 i}, C_{3 i}$, $i=1,2, \ldots, m$, there are different methods like Galerkin's method, Ritz method, least squares method, and collocation method. One can apply the method of least squares as follows:

$$
\begin{gathered}
J_{1}\left(C_{1 i}\right)=\int_{0}^{t} \int_{\Omega} R_{1}^{2}\left(x, t ; C_{1 i}\right) d x d t \\
J_{2}\left(C_{2 i}\right)=\int_{0}^{t} \int_{\psi} R_{2}^{2}\left(x, t ; C_{2 i}\right) d x d t \\
J_{3}\left(C_{3 i}\right)=\int_{0}^{t} \int_{\phi} R_{3}^{2}\left(x, t ; C_{3 i}\right) d x d t \\
\frac{\partial J_{1}}{\partial C_{11}}=\frac{\partial J_{1}}{\partial C_{12}}=\cdots \frac{\partial J_{1}}{\partial C_{1 m}}=\frac{\partial J_{2}}{\partial C_{21}}=\frac{\partial J_{2}}{\partial C_{22}}=\cdots \frac{\partial J_{2}}{\partial C_{2 m}} \\
=\frac{\partial J_{3}}{\partial C_{31}}=\frac{\partial J_{3}}{\partial C_{32}}=\cdots=\frac{\partial J_{3}}{\partial C_{3 m}}=0
\end{gathered}
$$

The $m$ th order approximate solution can be obtained by these constants so-obtained. The more general auxiliary functions $H_{1}(p), H_{2}(p), H_{3}(p)$ are useful for convergence, which depends upon constants $C_{11}, C_{12}, C_{21}, C_{22}, C_{31}, C_{32}, \ldots$, can be optimally identified by (17), and is useful in error minimization.

\section{Application of Extended OHAM to Coupled Schrödinger-KdV Equation}

To demonstrate the effectiveness of the extended OHAM for coupled Schrödinger- KdV equation taken from [6], we have

$$
\begin{gathered}
\frac{\partial \beta(x, t)}{\partial t}-\frac{\partial^{2} \eta(x, t)}{\partial x^{2}}-\eta(x, t) \mu(x, t)=0, \\
\frac{\partial \eta(x, t)}{\partial t}+\frac{\partial^{2} \beta(x, t)}{\partial x^{2}}+\beta(x, t) \mu(x, t)=0, \\
\frac{\partial \mu(x, t)}{\partial t}+6 \mu(x, t) \frac{\partial \mu(x, t)}{\partial x}+\frac{\partial^{3} \mu(x, t)}{\partial x^{3}} \\
-2 \beta(x, t) \frac{\partial \mu(x, t)}{\partial x}-2 \eta(x, t) \frac{\partial \eta(x, t)}{\partial x}=0,
\end{gathered}
$$

with boundary conditions

$$
\begin{gathered}
\beta(x, 0)=\cos (x), \\
\eta(x, 0)=\sin (x), \\
\mu(x, 0)=\frac{3}{4} .
\end{gathered}
$$

The exact solution of (19) for $-3 \leq x \leq 3$ and $0 \leq t \leq 1$ is given by

$$
\begin{gathered}
\beta(x, t)=\cos \left(x+\frac{t}{4}\right), \\
\eta(x, t)=\sin \left(x+\frac{t}{4}\right), \\
\mu(x, t)=\frac{3}{4} .
\end{gathered}
$$

Applying the extended OHAM technique discussed in Section 2,

$$
\begin{aligned}
& (1-p) \frac{\partial \beta(x, t)}{\partial t} \\
& -H_{1}(p)\left[\frac{\partial \beta(x, t)}{\partial t}-\frac{\partial^{2} \eta(x, t)}{\partial x^{2}}-\eta(x, t) \mu(x, t)\right]=0, \\
& (1-p) \frac{\partial \eta(x, t)}{\partial t} \\
& -H_{2}(p)\left[\frac{\partial \eta(x, t)}{\partial t}+\frac{\partial^{2} \beta(x, t)}{\partial x^{2}}+\beta(x, t) \mu(x, t)\right]=0, \\
& (1-p) \frac{\partial \mu(x, t)}{\partial t}\left[\frac{\partial \mu(x, t)}{\partial t}+6 \mu(x, t) \frac{\partial \mu(x, t)}{\partial x}+\frac{\partial^{3} \mu(x, t)}{\partial x^{3}}\right. \\
& -H_{3}(p)\left[\frac{\partial \eta(x, t)}{\partial x}\right]
\end{aligned}
$$$$
=0 \text {. }
$$ 
TABLE 1: Absolute error of OHAM solution $\beta(x, t)$ corresponding to the exact solution.

\begin{tabular}{lccccc}
\hline$x$ & $t=0.5$ & $t=0.2$ & $t=0.1$ & $t=0.01$ & $t=0.001$ \\
\hline-3 & $4.37771 \times 10^{-3}$ & $8.5962 \times 10^{-4}$ & $3.51139 \times 10^{-4}$ & $6.29194 \times 10^{-5}$ & $6.57034 \times 10^{-6}$ \\
-2 & $1.83163 \times 10^{-2}$ & $8.0058 \times 10^{-3}$ & $4.12582 \times 10^{-3}$ & $4.24051 \times 10^{-4}$ & $4.25219 \times 10^{-5}$ \\
-1 & $2.41704 \times 10^{-2}$ & $8.5651 \times 10^{-3}$ & $4.10724 \times 10^{-3}$ & $3.95312 \times 10^{-4}$ & $3.93790 \times 10^{-5}$ \\
0 & $7.80233 \times 10^{-3}$ & $1.2497 \times 10^{-3}$ & $3.12484 \times 10^{-4}$ & $3.12500 \times 10^{-6}$ & $3.12500 \times 10^{-8}$ \\
1 & $1.57391 \times 10^{-2}$ & $7.2147 \times 10^{-3}$ & $3.76957 \times 10^{-3}$ & $3.91935 \times 10^{-4}$ & $3.93452 \times 10^{-5}$ \\
2 & $2.48101 \times 10^{-2}$ & $9.0460 \times 10^{-3}$ & $4.38590 \times 10^{-3}$ & $4.26652 \times 10^{-4}$ & $4.25479 \times 10^{-5}$ \\
3 & $1.10708 \times 10^{-3}$ & $2.5600 \times 10^{-3}$ & $9.69852 \times 10^{-4}$ & $6.91069 \times 10^{-5}$ & $6.63222 \times 10^{-6}$ \\
\hline
\end{tabular}

TABLE 2: Absolute error of OHAM solution $\eta(x, t)$ corresponding to the exact solution.

\begin{tabular}{lccccc}
\hline$x$ & $t=0.5$ & $t=0.2$ & $t=0.1$ & $t=0.01$ & $t=0.001$ \\
\hline-3 & $1.33126 \times 10^{-4}$ & $1.6026 \times 10^{-4}$ & $2.83128 \times 10^{-5}$ & $1.39272 \times 10^{-6}$ & $1.79217 \times 10^{-7}$ \\
-2 & $7.19141 \times 10^{-3}$ & $1.12962 \times 10^{-3}$ & $2.77505 \times 10^{-4}$ & $2.07074 \times 10^{-6}$ & $4.87727 \times 10^{-8}$ \\
-1 & $6.4398 \times 10^{-3}$ & $1.06041 \times 10^{-3}$ & $2.71500 \times 10^{-4}$ & $3.63037 \times 10^{-6}$ & $1.26513 \times 10^{-7}$ \\
0 & $2.32524 \times 10^{-4}$ & $1.62664 \times 10^{-5}$ & $1.59445 \times 10^{-5}$ & $1.85225 \times 10^{-6}$ & $1.85483 \times 10^{-7}$ \\
1 & $6.69107 \times 10^{-3}$ & $1.04283 \times 10^{-3}$ & $2.54331 \times 10^{-4}$ & $1.62882 \times 10^{-6}$ & $7.39210 \times 10^{-8}$ \\
2 & $6.99788 \times 10^{-3}$ & $1.14315 \times 10^{-3}$ & $2.90776 \times 10^{-4}$ & $3.61236 \times 10^{-6}$ & $1.05604 \times 10^{-7}$ \\
3 & $8.70868 \times 10^{-4}$ & $1.92467 \times 10^{-4}$ & $5.98826 \times 10^{-5}$ & $2.27472 \times 10^{-6}$ & $1.88037 \times 10^{-7}$ \\
\hline
\end{tabular}

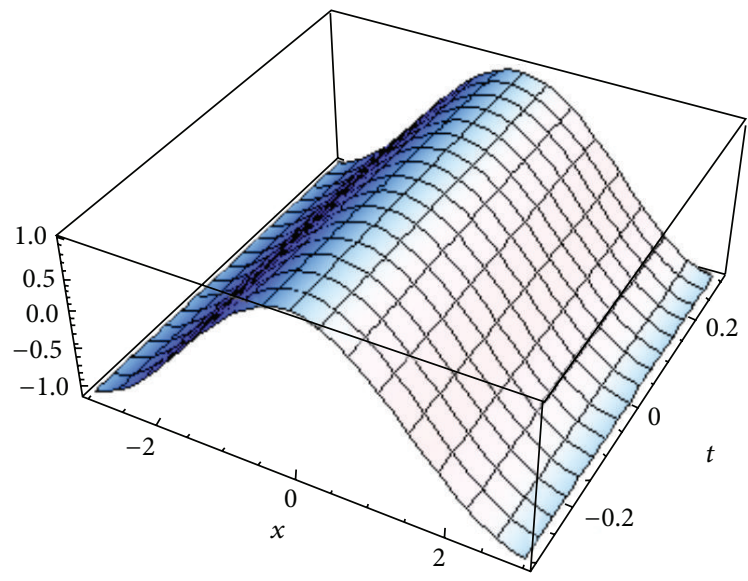

Figure 1: 3D, OHAM solution of $\beta(x, t)$ at $t=0.1$.

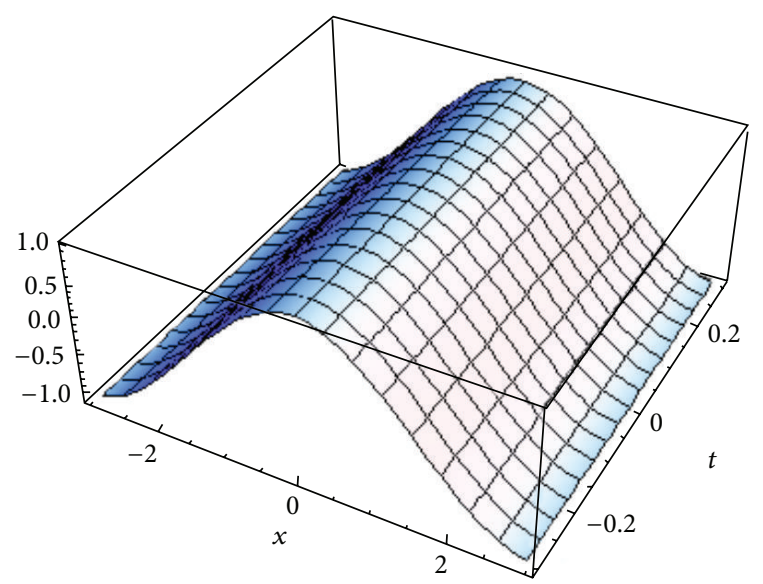

Figure 2: 3D, exact solution of $\beta(x, t)$ at $t=0.1$.

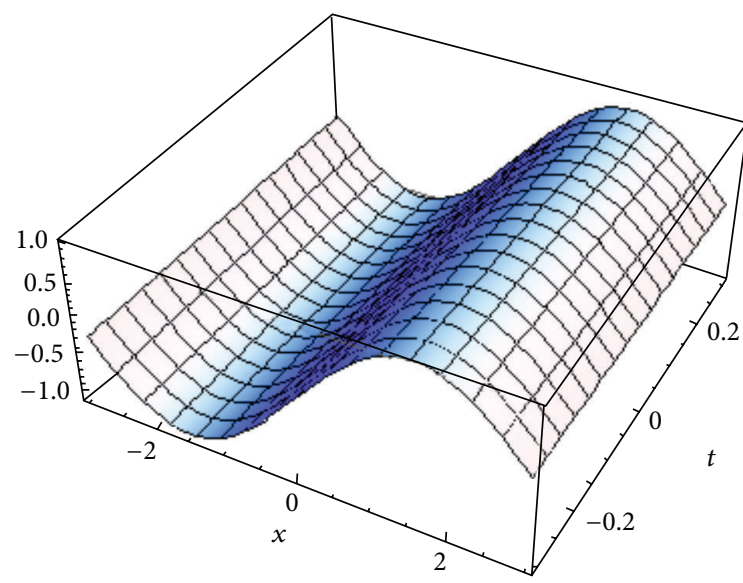

FIgURE 3: 3D, OHAM solution of $\eta(x, t)$ at $t=0.1$.

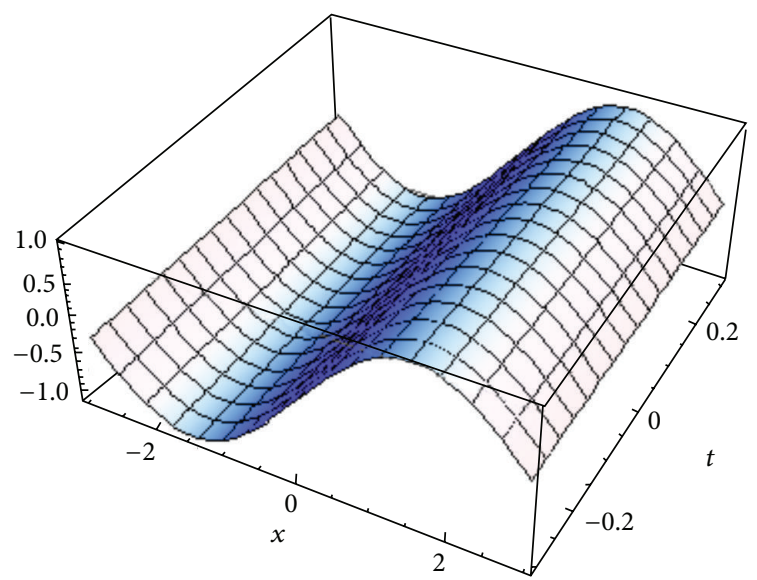

FIgURE 4: 3D, exact solution of $\eta(x, t)$ at $t=0.1$. 
TABLE 3: Comparison of $\mu(x, t)$ solutions obtained by OHAM to the exact solution.

\begin{tabular}{lcc}
\hline$x$ & OHAM solution & Exact solution \\
\hline-3 & $3 / 4$ & $3 / 4$ \\
-2 & $3 / 4$ & $3 / 4$ \\
-1 & $3 / 4$ & $3 / 4$ \\
0 & $3 / 4$ & $3 / 4$ \\
1 & $3 / 4$ & $3 / 4$ \\
2 & $3 / 4$ & $3 / 4$ \\
3 & $3 / 4$ & $3 / 4$ \\
\hline
\end{tabular}

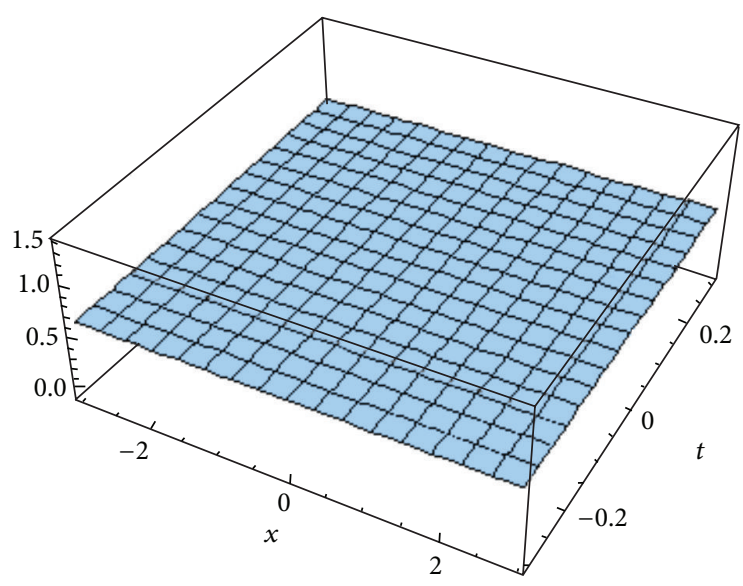

Figure 5: 3D, OHAM solution of $\mu(x, t)$ at $t=0.1$.

We consider

$$
\begin{gathered}
\beta=\beta_{0}+p \beta_{1}+p^{2} \beta_{2}, \quad \eta=\eta_{0}+p \eta_{1}+p^{2} \eta_{2}, \\
\mu=\mu_{0}+p \mu_{1}+p^{2} \mu_{2}, \\
H_{1}(p)=p C_{11}+p^{2} C_{12}, \\
H_{2}(p)=p C_{21}+p^{2} C_{22}, \quad H_{3}(p)=p C_{31}+p^{2} C_{32} .
\end{gathered}
$$

Zeroth Order System. Consider

$$
\frac{\partial \beta_{0}}{\partial t}=0, \quad \frac{\partial \eta_{0}}{\partial t}=0, \quad \frac{\partial \mu_{0}}{\partial t}=0,
$$

with initial conditions

$$
\begin{gathered}
\beta_{0}(x, 0)=\cos (x), \\
\eta_{0}(x, 0)=\sin (x), \\
\mu_{0}(x, 0)=\frac{3}{4} .
\end{gathered}
$$

Its solution is

$$
\begin{gathered}
\beta_{0}(x, t)=\cos (x), \\
\eta_{0}(x, t)=\sin (x), \\
\mu_{0}(x, t)=\frac{3}{4} .
\end{gathered}
$$

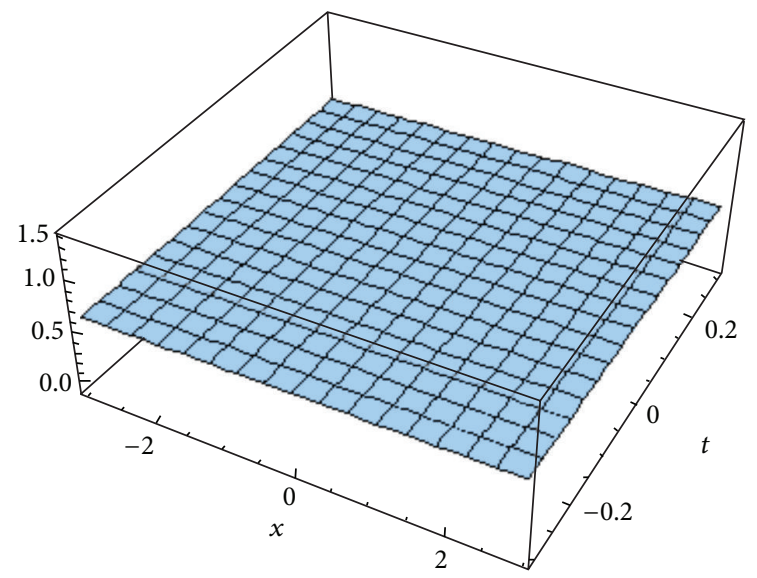

Figure 6: 3D, exact solution of $\mu(x, t)$ at $t=0.1$.

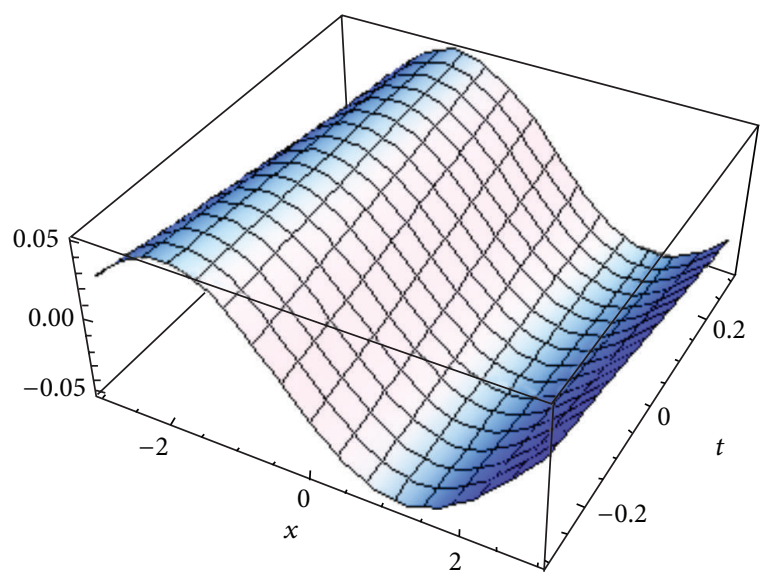

FIgURE 7: 3D, residual of $\beta(x, t)$ at $t=0.1$.

First Order System. Consider

$$
\begin{aligned}
\frac{\partial \beta_{1}(x, t)}{\partial t}= & \left(1+C_{11}\right) \frac{\partial \beta_{0}}{\partial t}-C_{11} \eta_{0} \mu_{0}-C_{11} \frac{\partial^{2} \eta_{0}}{\partial x^{2}} \\
\frac{\partial \eta_{1}(x, t)}{\partial t}= & \left(1+C_{21}\right) \frac{\partial \eta_{0}}{\partial t}+C_{21} \beta_{0} \mu_{0}+C_{21} \frac{\partial^{2} \beta_{0}}{\partial x^{2}} \\
\frac{\partial \mu_{1}(x, t)}{\partial t}= & \left(1+C_{31}\right) \frac{\partial \mu_{0}}{\partial t} \\
& -2 C_{31}\left(\beta_{0} \frac{\partial \beta_{0}}{\partial x}+\eta_{0} \frac{\partial \eta_{0}}{\partial x}-3 \mu_{0} \frac{\partial \mu_{0}}{\partial x}\right) \\
& +C_{31} \frac{\partial^{3} \mu_{0}}{\partial x^{3}}
\end{aligned}
$$

with

$$
\beta_{1}(x, 0)=0, \quad \eta_{1}(x, 0)=0, \quad \mu_{1}(x, 0)=0 .
$$




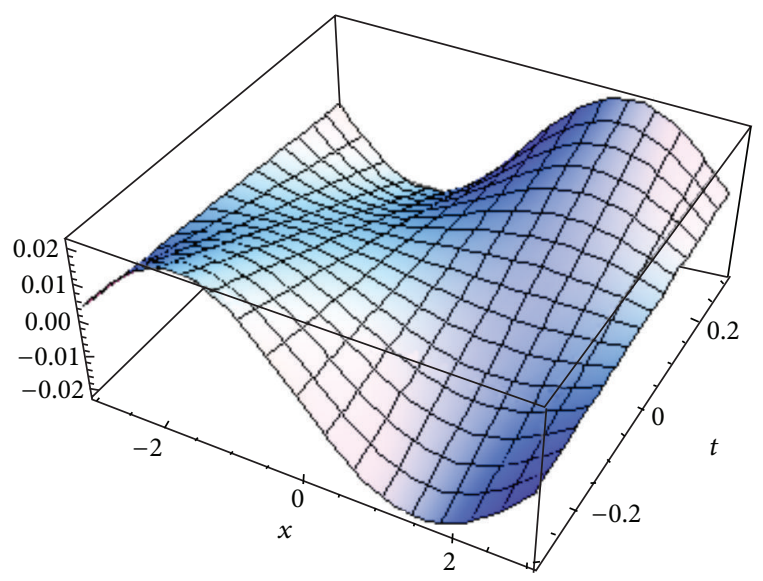

Figure $8: 3 \mathrm{D}$, residual of $\eta(x, t)$ at $t=0.1$.

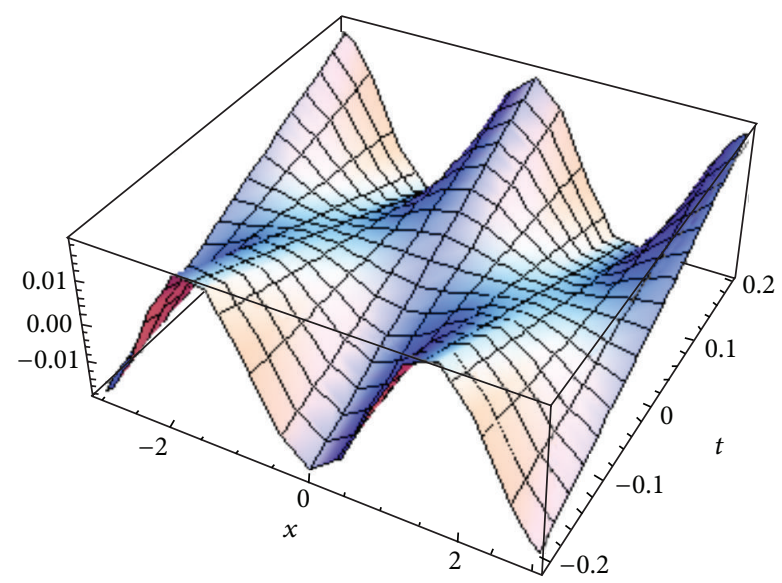

FIgURE 9: 3D, residual of $\mu(x, t)$ at $t=0.1$.

Its solution is

$$
\begin{gathered}
\beta_{1}\left(x, t, C_{11}\right)=\frac{C_{11}}{4} t \sin x, \\
\eta_{1}\left(x, t, C_{21}\right)=-\frac{C_{21}}{4} t \cos x, \\
\mu_{1}\left(x, t, C_{31}\right)=0 .
\end{gathered}
$$

Second Order System. Consider

$$
\begin{gathered}
\frac{\partial \beta_{2}(x, t)}{\partial t}=\left[\left(1+C_{11}\right) \frac{\partial \beta_{1}}{\partial t}-C_{11}\left(\eta_{1} \mu_{0}+\eta_{0} \mu_{1}\right)\right. \\
\left.+C_{12}\left(\beta_{0}-\eta_{0} \mu_{0}\right)-C_{12} \frac{\partial^{2} \eta_{0}}{\partial x^{2}}-C_{11} \frac{\partial^{2} \eta_{1}}{\partial x^{2}}\right], \\
\frac{\partial \eta_{2}(x, t)}{\partial t}=\left[\begin{array}{c}
\left(1+C_{21}\right) \frac{\partial \eta_{0}}{\partial t}+C_{21}\left(\beta_{1} \mu_{0}+\beta_{0} \mu_{1}\right) \\
\left.+C_{22}\left(\eta_{0}+\beta_{0} \mu_{0}\right)+C_{21} \frac{\partial^{2} \beta_{1}}{\partial x^{2}}+C_{22} \frac{\partial^{2} \mu_{0}}{\partial x^{2}}\right]
\end{array}\right.
\end{gathered}
$$

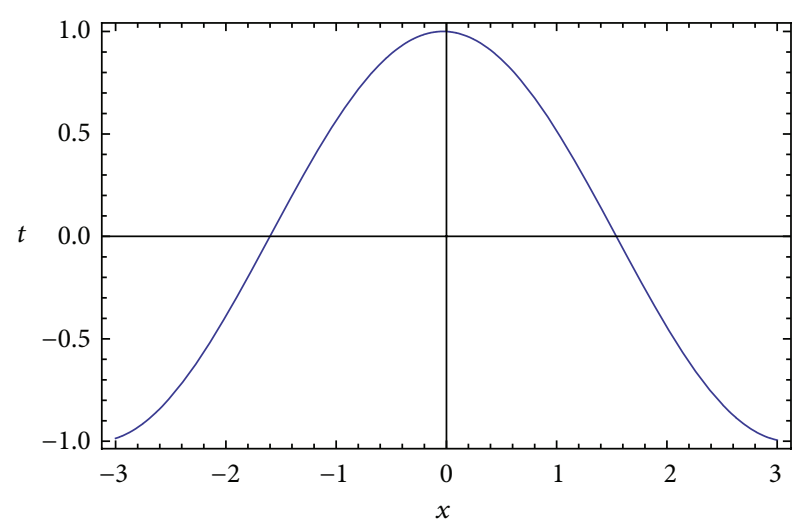

Figure 10: 2D, OHAM solution of $\beta(x, t)$ at $t=0.1$.

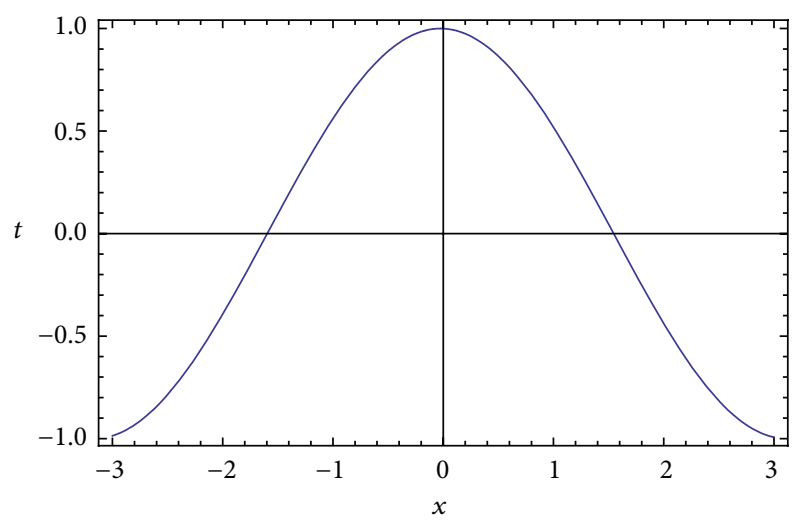

Figure 11: 2D, exact solution of $\beta(x, t)$ at $t=0.1$.

$$
\begin{aligned}
\frac{\partial \mu_{2}(x, t)}{\partial t}=\left[\left(1+C_{31}\right) \frac{\partial \mu_{1}}{\partial t}-2 C_{31}\right. & \\
& \times\left(\beta_{1} \frac{\partial \beta_{0}}{\partial x}+\beta_{0} \frac{\partial \beta_{1}}{\partial x}+\eta_{1} \frac{\partial \eta_{0}}{\partial x}+\eta_{0} \frac{\partial \eta_{1}}{\partial x}\right. \\
& \left.-3 \mu_{1} \frac{\partial \mu_{0}}{\partial x}-3 \mu_{0} \frac{\partial \mu_{1}}{\partial x}\right) \\
& -2 C_{32}\left(\beta_{0} \frac{\partial \beta_{0}}{\partial x}+\eta_{0} \frac{\partial \eta_{0}}{\partial x}-3 \mu_{0} \frac{\partial \mu_{0}}{\partial x}\right) \\
& \left.+C_{31} \frac{\partial \mu_{0}}{\partial x}+C_{32} \frac{\partial^{3} \mu_{0}}{\partial x^{3}}+C_{31} \frac{\partial^{3} \mu_{1}}{\partial x^{3}}\right]
\end{aligned}
$$

with

$$
\beta_{2}(x, 0)=0, \quad \eta_{2}(x, 0)=0, \quad \mu_{2}(x, 0)=0 .
$$

The solution of second order system is

$$
\begin{aligned}
\beta_{2}\left(x, t, C_{11}, C_{12}\right)=\frac{1}{32}( & 8 C_{11} t \sin x+8 C_{11}^{2} t \sin x \\
& \left.+8 C_{12} t \sin x-t^{2} C_{11} C_{21} \cos x\right),
\end{aligned}
$$




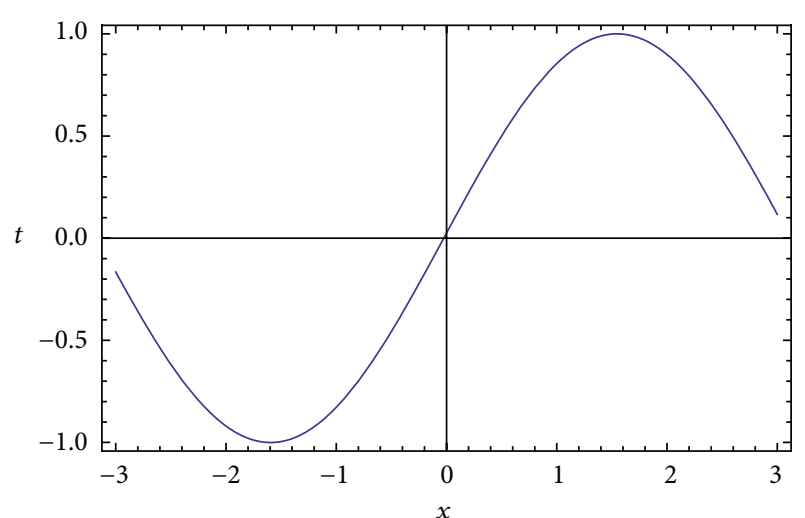

FIGURE 12: 2D, OHAM solution of $\eta(x, t)$ at $t=0.1$.

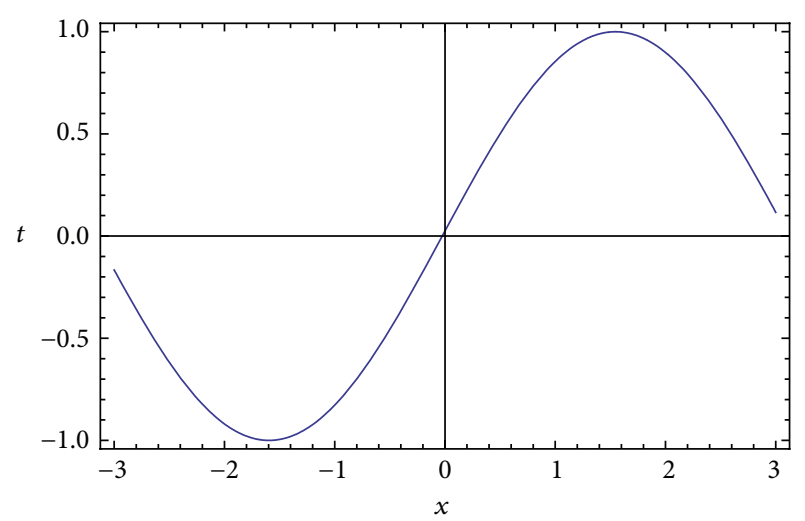

FIGURE 13: 2D, exact solution of $\eta(x, t)$ at $t=0.1$.

$$
\begin{aligned}
\eta_{2}\left(x, t, C_{21}, C_{22}\right)=-\frac{1}{32}( & 8 C_{21} \cos x+8 C_{21}^{2} t \cos x \\
& \left.+C_{11} C_{21} t^{2} \sin x+C_{22} t \cos x\right)
\end{aligned}
$$$$
\mu_{2}\left(x, t, C_{31}\right)=\frac{1}{4}\left(C_{21} C_{31} t^{2} \cos (2 x)-C_{11} C_{31} t^{2} \cos (2 x)\right) \text {. }
$$

Adding (26), (29), and (32), we obtain

$$
\begin{aligned}
\beta\left(x, t, C_{11}, C_{21}\right) & \\
= & \cos (x)+\frac{1}{4} C_{11} t \sin (x) \\
& +\frac{1}{32}\left(8 C_{11} t \sin x+8 C_{11}^{2} t \sin x\right. \\
& \left.+8 C_{12} t \sin x-t^{2} C_{11} C_{21} \cos x\right),
\end{aligned}
$$

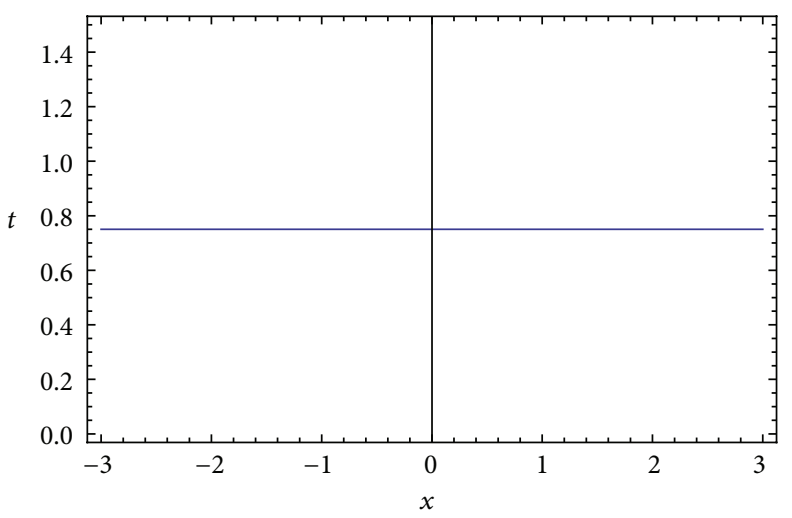

Figure 14: 2D, OHAM solution of $\mu(x, t)$ at $t=0.1$.

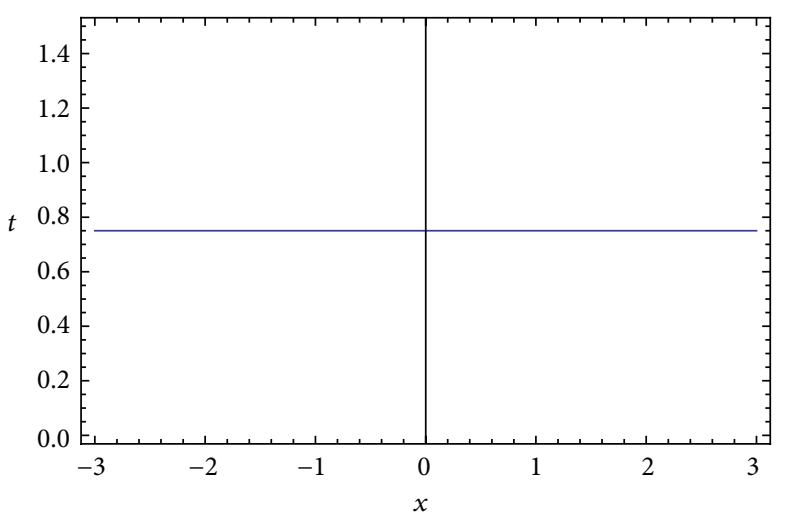

Figure 15: 2D, exact solution of $\mu(x, t)$ at $t=0.1$.

$$
\begin{gathered}
\eta\left(x, t, C_{21}, C_{22}\right) \\
=\sin (x)-\frac{1}{4} C_{21} t \sin (x) \\
\quad-\frac{1}{32}\left(8 C_{21} \cos x+8 C_{21}^{2} t \cos x\right. \\
\left.\quad+C_{11} C_{21} t^{2} \sin x+C_{22} t \cos x\right), \\
\mu\left(x, t, C_{31}\right)=\frac{3}{4}+\frac{1}{4}\left(C_{21} C_{31} t^{2} \cos (2 x)\right. \\
\left.\quad-C_{11} C_{31} t^{2} \cos (2 x)\right) .
\end{gathered}
$$

For the calculation of the constants $C_{11}, C_{12}, C_{21}, C_{22}$, and $C_{31}$ using (33) in (19) and applying the method of least square mentioned in (16)-(17) by taking, we get

$$
\begin{gathered}
C_{11}=-3.041182429907255 \times 10^{-14}, \\
C_{12}=-1.1871110474593864 \\
C_{21}=-3.041182429907255 \times 10^{-14}, \\
C_{22}=-0.999258572471839, \\
C_{31}=-8.101774168020832 \times 10^{-15} .
\end{gathered}
$$




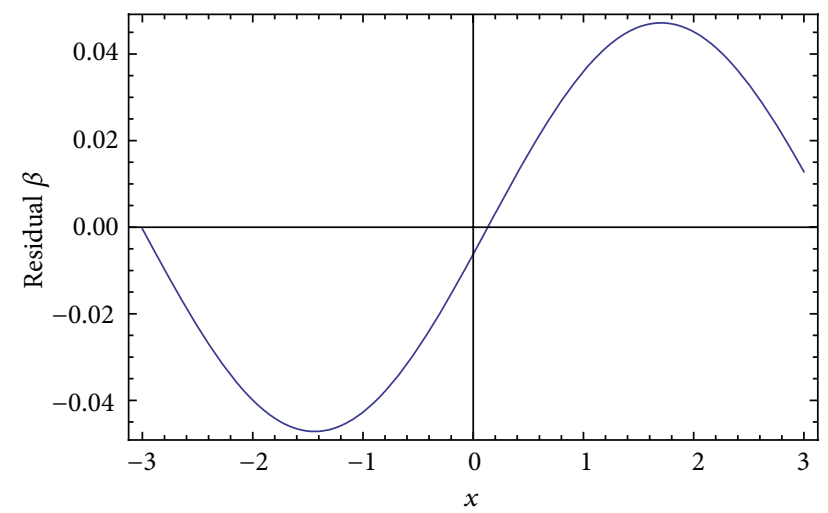

Residual $\beta$

FIGURE 16: 2D, residual of $\beta(x, t)$ at $t=0.1$.

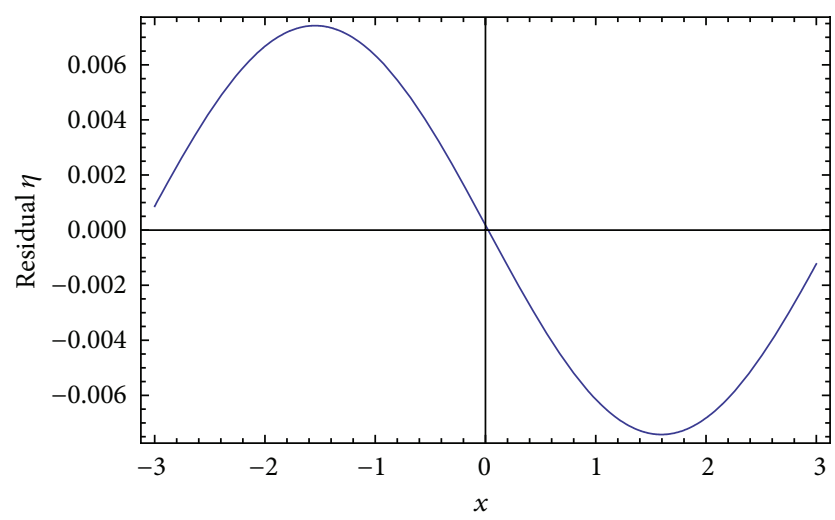

- Residual $\eta$

Figure 17: 2D, residual of $\eta(x, t)$ at $t=0.1$.

$$
\begin{aligned}
& \beta(x, t)=\left[\cos (x)-7.602956074768137 \times 10^{-15} t \sin (x)\right. \\
& +\frac{1}{32}\left(-9.248790571976596 \times 10^{-28} t^{2} \cos (x)\right. \\
& -9.496888379675335 t \sin (x))], \\
& \eta(x, t)=\left[7.602956074768137 \times 10^{-15} t \cos (x)+\sin (x)\right. \\
& +\frac{1}{32}(7.994064457977714 t \cos (x) \\
& \left.\left.-9.248790571976596 \times 10^{-28} t^{2} \sin (x)\right)\right] \text {, } \\
& \mu(x, t)=\left[\frac{3}{4}\right]
\end{aligned}
$$

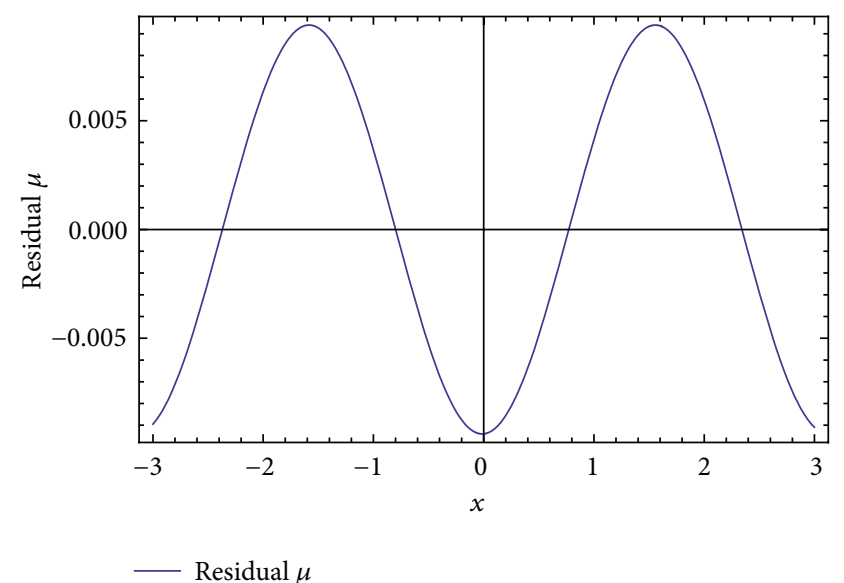

FIgURE 18: 2D, residual of $\mu(x, t)$ at $t=0.1$.

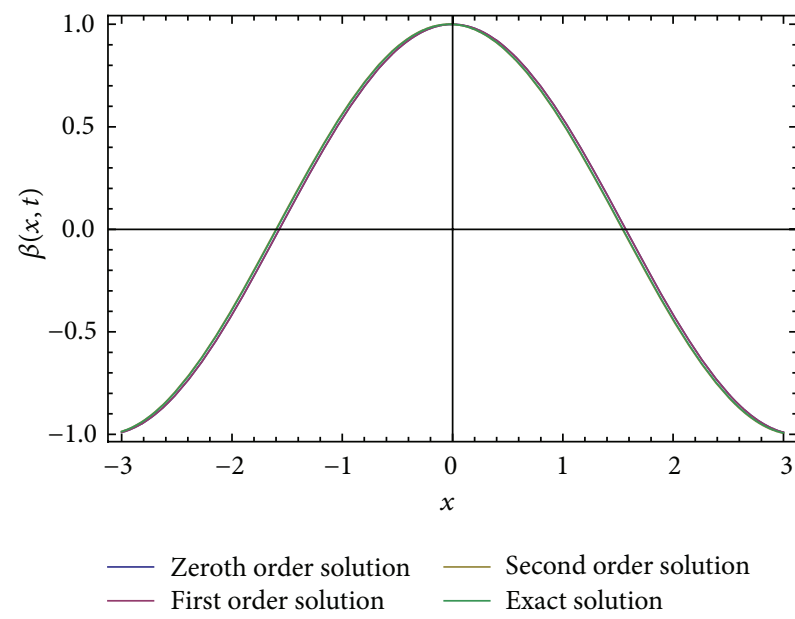

FIGURE 19: 2D, comparison of ordered solutions for $\beta(x, t)$ at $t=0.1$.

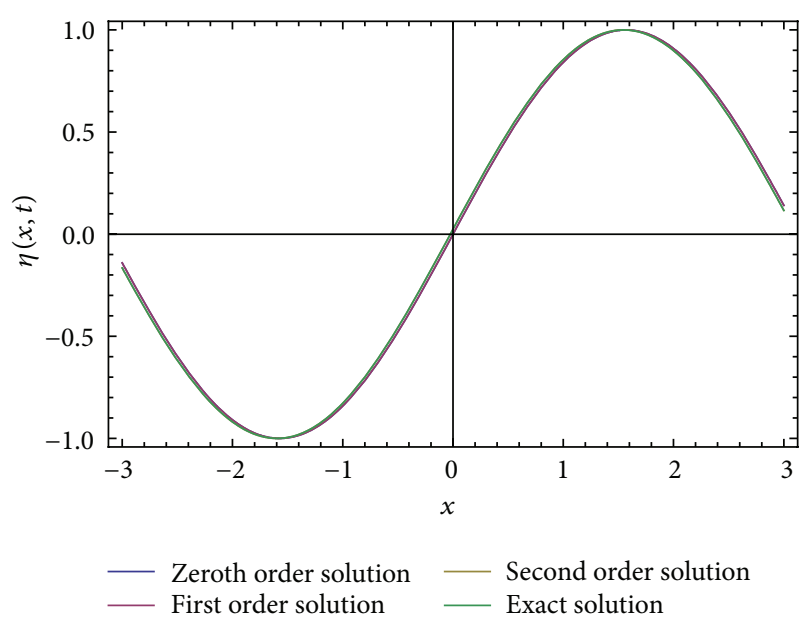

FIgURE 20: 2D, comparison of ordered solutions for $\eta(x, t)$ at $t=0.1$. 


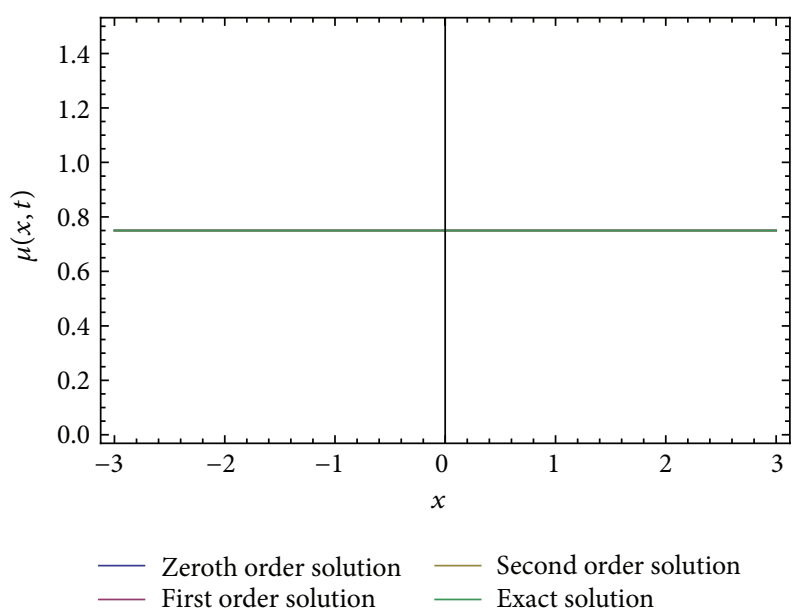

Figure 21: 2D, comparison of ordered solutions for $\mu(x, t)$ at $t=0.1$.

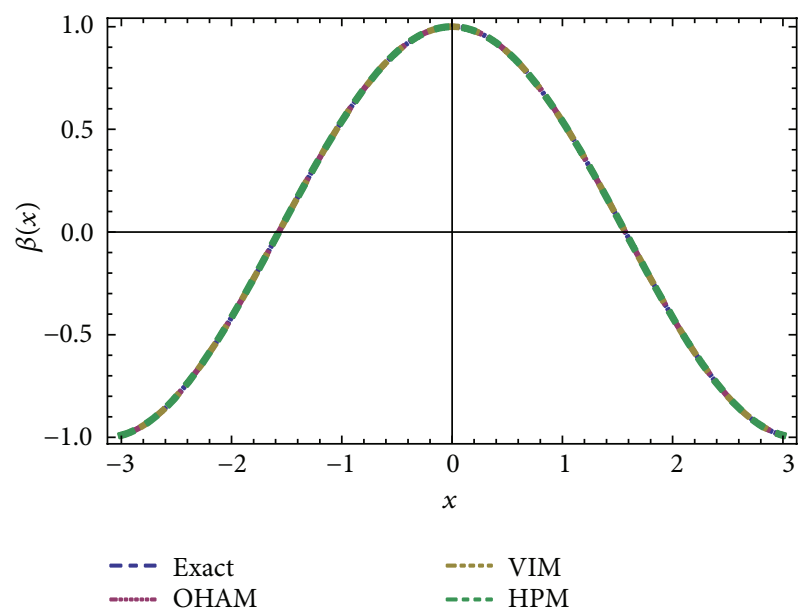

Figure 22: 2D, comparison of OHAM, VIM, HPM, and exact solutions for $\beta(x, t)$ at $t=0.1$.

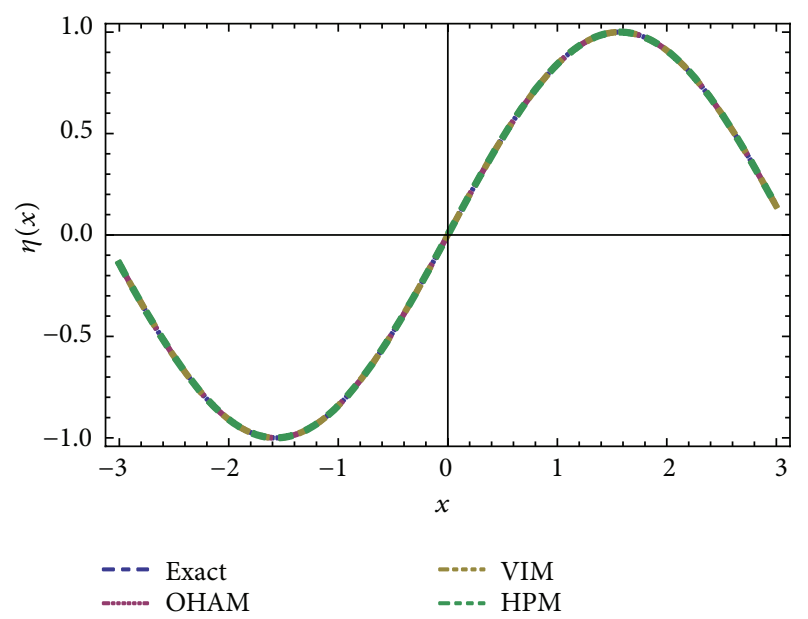

FIgURE 23: 2D, comparison of OHAM, VIM, HPM, and exact solutions for $\eta(x, t)$ at $t=0.1$.

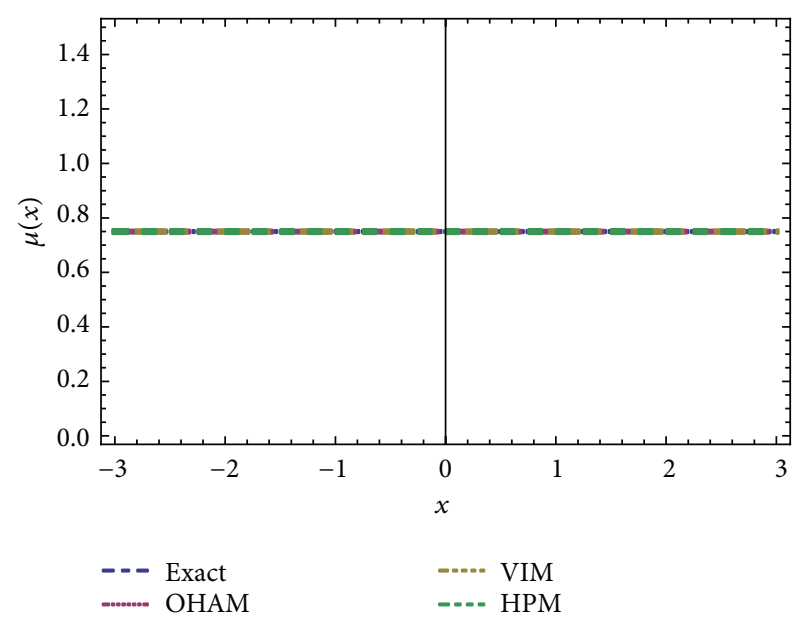

FIgURE 24: 2D, comparison of OHAM, VIM, HPM, and exact solutions for $\mu(x, t)$ at $t=0.1$.

The solution obtained by variational iteration method [6] is as follows:

$$
\begin{gathered}
\beta(x, t)=\cos (x)-\frac{1}{4} t \sin (x)-\frac{1}{32} t^{2} \cos (x), \\
\eta(x, t)=\sin (x)+\frac{1}{4} t \cos (x)-\frac{1}{32} t^{2} \sin (x), \\
\mu(x, t)=\frac{3}{4} .
\end{gathered}
$$

The solution obtained by homotopy perturbation method [2] is as follows:

$$
\begin{aligned}
& \beta(x, t)= \cos (x)-\frac{1}{4} t \sin (x) \\
&-\frac{1}{32} t^{2} \cos (x)+\frac{1}{384} t^{3} \sin (x), \\
& \eta(x, t)= \sin (x)+\frac{1}{4} t \cos (x) \\
&-\frac{1}{32} t^{2} \sin (x)-\frac{1}{384} t^{3} \cos (x), \\
& \mu(x, t)=\frac{3}{4} .
\end{aligned}
$$

\section{Results and Discussions}

The extended mathematical theory presented in Section 2 gives highly accurate solutions for the BVP presented in Section 3. We have used Mathematica 7 for most of our computational work. In Tables 1 and 2, the absolute errors of extended OHAM results corresponding to the exact solution of the problem given in (19) are given for $\beta(x, t), \eta(x, t)$ at $t=0.5,0.2,0.1,0.01,0.001$. In Table 3 , the extended OHAM results are compared with exact solution for $\mu(x, t)$. In Figures 1, 2, 3, 4, 5, and 6, 3D plots of the results obtained by extended OHAM and exact solutions are plotted for $\beta(x, t), \eta(x, t), \mu(x, t)$ at fixed value of $t=0.1$. While the $3 \mathrm{D}$ 
plots of the residuals at $t=0.1$ are given in Figures 7, 8, and 9, Figures $10,11,12,13,14$, and 15 show the $2 \mathrm{D}$ plots of extended OHAM and exact solutions for $\beta(x, t), \eta(x, t), \mu(x, t)$ at $t=$ 0.1 . The $2 \mathrm{D}$ residual plots are given in Figures 16,17 , and 18 at $t=0.1$. In Figures 19, 20, and 21 the 2D plots of ordered solutions are given at $t=0.1$ for $\beta(x, t), \eta(x, t), \mu(x, t)$. The extended OHAM solutions are compared with VIM and HPM solution in 2D, Figures 22, 23, and 24. Here we observed from Figures 19-21 that the extended OHAM solution converges rapidly with the increase in the order of approximation and produced better results. From Figures 2224 it is evident that the extended OHAM results are nearly identical to the exact, VIM, and HPM solutions. Here the results are very consistent with the decreasing time as evident from Tables 1-2.

\section{Conclusion}

In this paper, we have seen the effectiveness of extended OHAM to coupled Schrödinger-KdV equation. By applying the basic idea of extended OHAM to coupled Schrödinger$\mathrm{KdV}$ equation, we found it simpler in applicability and more convenient to control convergence and that it involved less computational overhead. Therefore, extended OHAM shows its validity and great potential for the solution of nonlinear system of PDEs problems in science and engineering.

\section{Conflict of Interests}

The authors declare that there is no conflict of interests regarding the publication of this paper.

\section{References}

[1] H. Wang, "Numerical studies on the split-step finite difference method for nonlinear Schrödinger equations," Applied Mathematics and Computation, vol. 170, no. 1, pp. 17-35, 2005.

[2] S. Küçükarslan, "Homotopy perturbation method for coupled Schrödinger-KdV equation," Nonlinear Analysis: Real World Applications, vol. 10, no. 4, pp. 2264-2271, 2009.

[3] D. Bai and L. Zhang, "Numerical studies on a novel splitstep quadratic B-spline finite element method for the coupled Schrödinger-KdV equations," Communications in Nonlinear Science and Numerical Simulation, vol. 16, no. 3, pp. 1263-1273, 2011.

[4] E. Fan and Y. C. Hon, "Applications of extended tanh method to "special" types of nonlinear equations," Applied Mathematics and Computation, vol. 141, no. 2-3, pp. 351-358, 2003.

[5] D. Kaya and S. M. El-Sayed, "On the solution of the coupled Schrödinger-KdV equation by the decomposition method," Physics Letters A, vol. 313, no. 1-2, pp. 82-88, 2003.

[6] A. Doosthoseini and H. Shahmohamadi, "Variational iteration method for solving coupled Schrödinger-KdV equation," Applied Mathematical Sciences, vol. 4, no. 17-20, pp. 823-837, 2010.

[7] A. K. Alomari, M. S. M. Noorani, and R. Nazar, "Comparison between the homotopy analysis method and homotopy perturbation method to solve coupled Schrodinger-KdV equation," Journal of Applied Mathematics and Computing, vol. 31, no. 1-2, pp. 1-12, 2009.
[8] L. Y. Qing, C. R. Jun, and G. H. Xia, "An element free Galerkin (EFG) method for numerical solution of coupled SchrödingerKdV equation," Chinese Physics B, vol. 22, Article ID 100204, 2013.

[9] A. Golbabai and A. Safdari-Vaighani, "A meshless method for numerical solution of the coupled Schrödinger-KdV equations," Computing, vol. 92, no. 3, pp. 225-242, 2011.

[10] V. Marinca, N. Herişanu, and I. Nemeş, "Optimal homotopy asymptotic method with application to thin film flow," Central European Journal of Physics, vol. 6, no. 3, pp. 648-653, 2008.

[11] N. Herişanu and V. Marinca, "Explicit analytical approximation to large-amplitude non-linear oscillations of a uniform cantilever beam carrying an intermediate lumped mass and rotary inertia," Meccanica, vol. 45, no. 6, pp. 847-855, 2010.

[12] V. Marinca, N. Herişanu, C. Bota, and B. Marinca, "An optimal homotopy asymptotic method applied to the steady flow of a fourth-grade fluid past a porous plate," Applied Mathematics Letters, vol. 22, no. 2, pp. 245-251, 2009.

[13] V. Marinca, N. Herisanu, and I. Nemes, "A new analytic approach to nonlinear vibration of an electrical machine," Proceedings of the Romanian Academy, vol. 9, pp. 229-236, 2008.

[14] V. Marinca and N. Herişanu, "Determination of periodic solutions for the motion of a particle on a rotating parabola by means of the optimal homotopy asymptotic method," Journal of Sound and Vibration, vol. 329, no. 9, pp. 1450-1459, 2010.

[15] S. Iqbal, M. Idrees, A. M. Siddiqui, and A. R. Ansari, "Some solutions of the linear and nonlinear Klein-Gordon equations using the optimal homotopy asymptotic method," Applied Mathematics and Computation, vol. 216, no. 10, pp. 2898-2909, 2010.

[16] H. Ullah, S. Islam, M. Idrees, and M. Arif, "Solution of boundary layer problems with heat transfer by optimal homotopy asymptotic method," Abstract and Applied Analysis, vol. 2013, Article ID 324869, 10 pages, 2013.

[17] H. Ullah, S. Islam, M. Idrees, and R. Nawaz, "Application of optimal homotopy asymptotic method to doubly wave solutions of the coupled Drinfel d-Sokolov-Wilson equations," Mathematical Problems in Engineering, vol. 2013, Article ID 362816, 8 pages, 2013. 


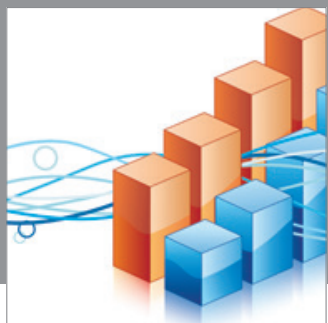

Advances in

Operations Research

mansans

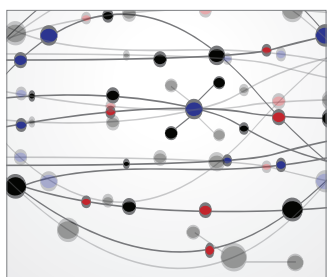

The Scientific World Journal
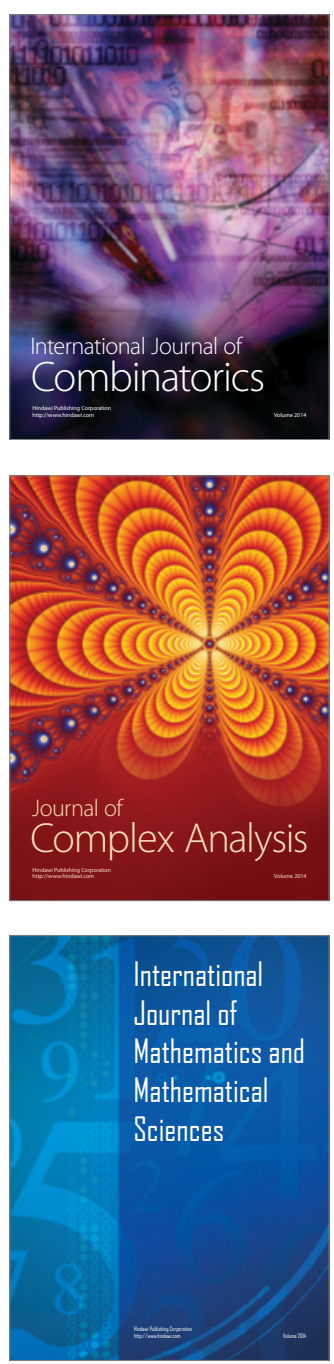
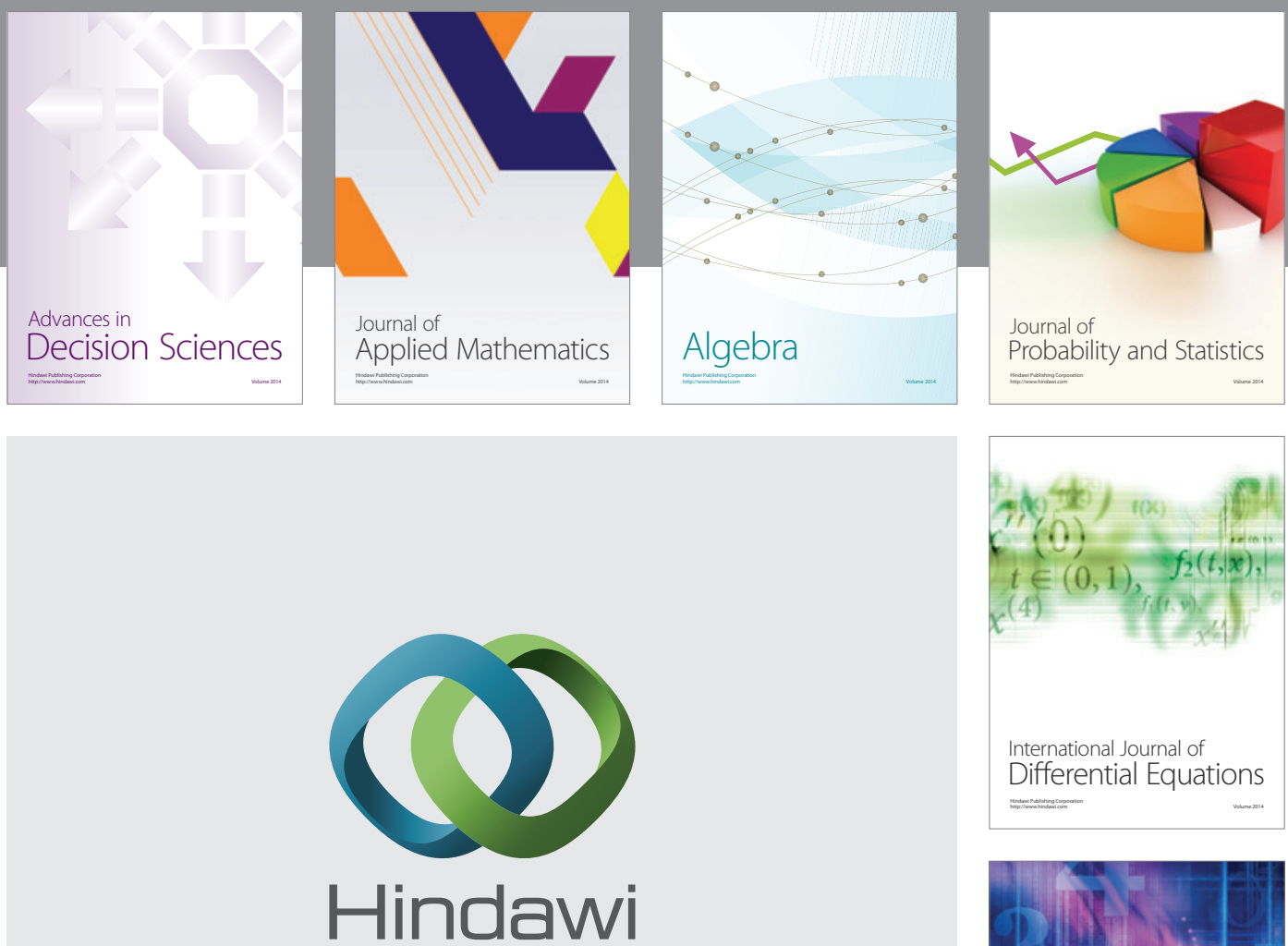

Submit your manuscripts at http://www.hindawi.com
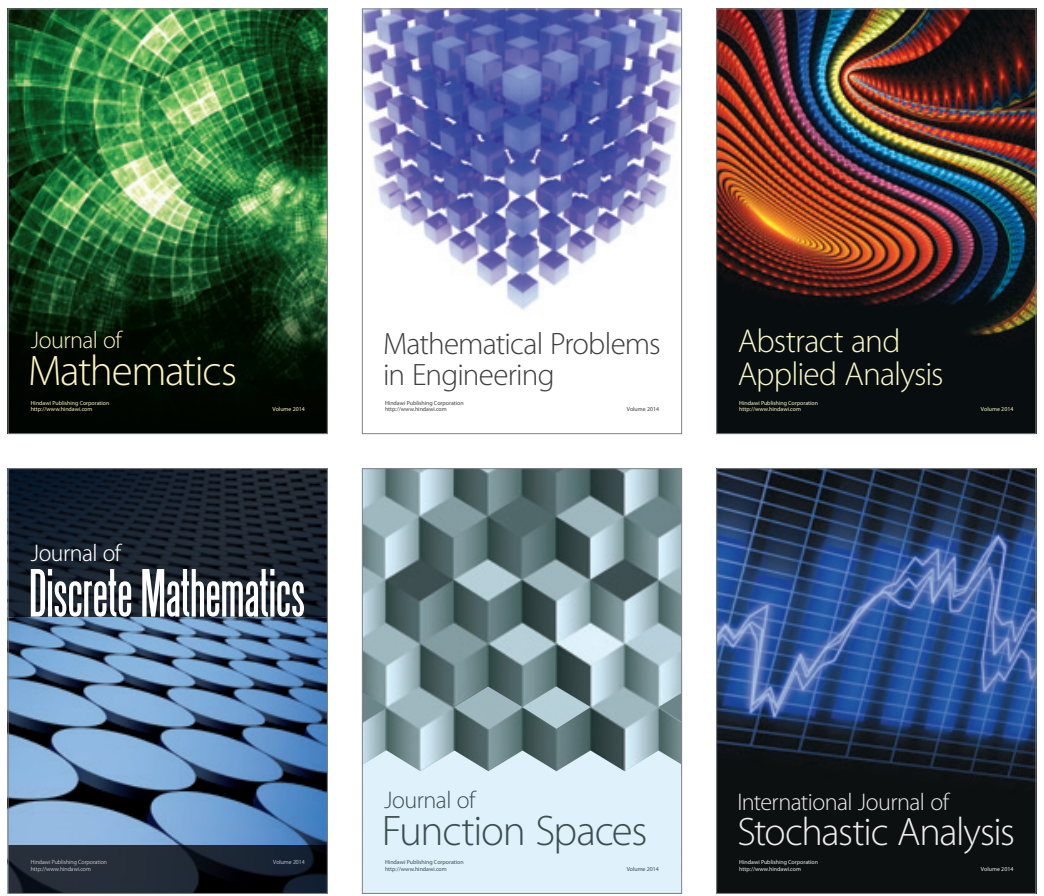

Journal of

Function Spaces

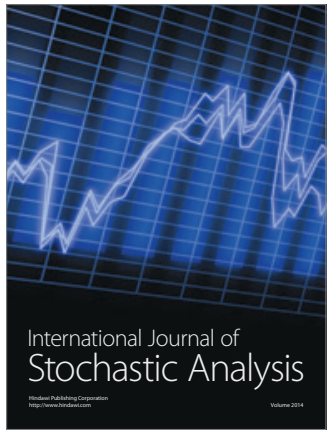

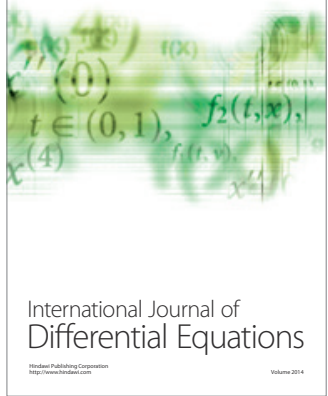
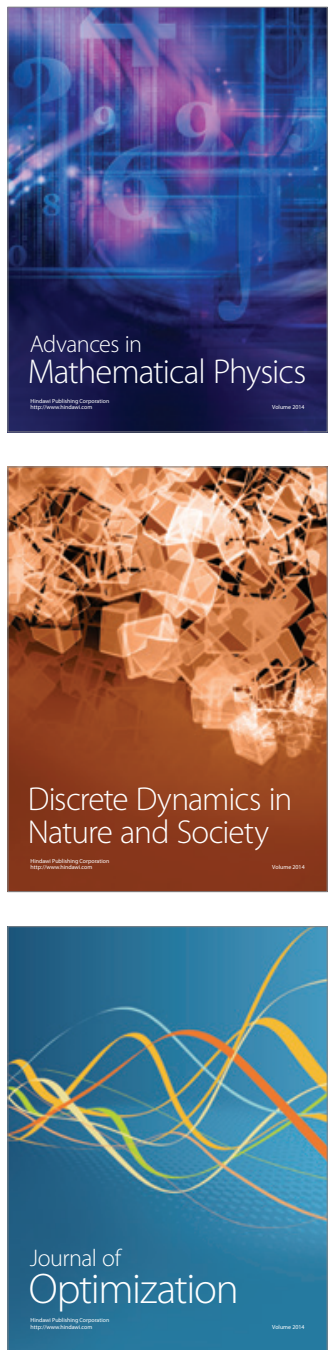